\title{
SAG/ROC2/Rbx2/Hrt2, a Component of SCF E3 Ubiquitin Ligase: Genomic Structure, a Splicing Variant, and Two Family Pseudogenes
}

\author{
MANJU SWAROOP, MARK GOSINK, ${ }^{1}$ and YI SUN
}

\begin{abstract}

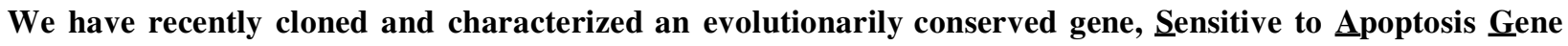
(SAG), which encodes a redox-sensitive antioxidant protein that protects cells from apoptosis induced by redox agents. The SAG protein was later found to be the second family member of $\mathrm{ROC} / \mathrm{Rbx} / \mathrm{Hrt}$, a component of the Skp1-cullin-F box protein (SCF) E3 ubiquitin ligase, being required for yeast growth and capable of promoting cell growth during serum starvation. Here, we report the genomic structure of the $S A G$ gene that consists of four exons and three introns. We also report the characterization of a $S A G$ splicing variant (SAG$v$ ), that contains an additional exon (exon 2; $264 \mathrm{bp}$ ) not present in wildtype SAG. The inclusion of exon 2 disrupts the SAG ORF and gives rise to a protein of 108 amino acids that contains the first 59 amino acids identical to SAG and a 49-amino acid novel sequence at the $C$ terminus. The entire RING-finger domain of SAG was not translated because of several inframe stop codons within the exon 2 . The SAG-v protein was expressed in multiple human tissues as well as cell lines, but at a much lower level than wildtype SAG. Unlike SAG, SAG-v was not able to rescue yeast cells from lethality in a ySAG knockout, nor did it bind to cullin-1 or have ligase activity, probably because of the lack of the RING-finger domain. Finally, we report the identification of two SAG family pseudogenes, SAGP1 and SAGP2, that share $36 \%$ or $47 \%$ sequence identity with ROC1/Rbx1/Hrt1 and $30 \%$ or $88 \%$ with SAG, respectively. Both genes are intronless with two inframe stop codons.
\end{abstract}

\section{INTRODUCTION}

$\mathbf{C}$ ELL CYCLE PROGRESSION is precisely regulated by timely synthesis and degradation of specific regulatory proteins. The major protein degradation pathway involves the ubiquitin/proteosome system. Ubiquitination of a target protein involves a multistep, well-defined set of reactions catalyzed by a cascade of enzyme families, including ubiquitin-activating enzyme E1, ubiquitin-conjugating enzymes E2, and ubiquitin ligases E3. Ubiquitin is first activated via binding to E1 through a thioester bond between a cysteine residue at the active site of E1 and the C-terminal glycine (G76) of ubiquitin. Activated ubiquitin in the E1-ubiquitin complex is then transferred to E2 that also forms a thioester bond between its active-site cysteine residue and G76 of ubiquitin. Finally, through the action of E3, ubiquitin is covalently attached to the target protein through an isopeptide bond between G76 of ubiquitin and the $\epsilon$ amino group of an internal lysine residue of the target protein. Through multiple reactions, a chain of ubiquitins is covalently attached to the substrates to form polyubiquitinated conjugates that are rapidly recognized and degraded by the $26 \mathrm{~S}$ proteosome (Ciechanover, 1998).

One of the well-defined E3 ubiquitin ligase complexes in eukaryotes that control cell cycle progression of $\mathrm{G}_{1} \rightarrow \mathrm{S}$ is $\mathrm{SCF}$ complex, consisting of $\underline{S} \mathrm{kp} 1$, cullins/Cdc53, and $\underline{\mathrm{F}}$-box proteins (Bai et al., 1996; Skowyra et al., 1997). The SCF complexes with a newly identified RING-H2 protein, ROC/Rbx/Hrt (Kamura et al., 1999; Ohta et al., 1999; Seol et al., 1999; Skowyra et al., 1999; Tan et al., 1999), to constitute an active E3 ubiquitin ligase that promotes ubiquitination and degradation of cyclin-dependent kinase inhibitors, such as p27 in mammals and Sic1 in yeast (Carrano et al., 1999; Feldman et al., 1997; Kamura et al., 1999; King et al., 1996; Skowyra et al., 1997; Sutterluty et al., 1999; Tsvetkov et al., 1999). 
We have also recently cloned and characterized an evolutionarily conserved RING finger protein, SAG (Sensitive to Apoptosis Gene), that is redox inducible and protects cells from apoptosis induced by redox agents (Duan et al., 1999; Sun, 1999, 2000; Swaroop et al., 1999). Sequence comparison has revealed that $\mathrm{SAG}$ is the second member of the $\mathrm{Rbx} / \mathrm{ROC} / \mathrm{Hrt}$ family (ROC2/Rbx2/Hrt2). The SAG/ROC/Rbx is required for yeast cell growth (Kamura et al., 1999; Ohta et al., 1999; Swaroop et al., 2000), can promote cell growth and S phase entry on serum starvation if overexpressed (Duan et al., 2001), and can inhibit tumor cell growth if repressed by antisense transfection (Huang et al., 2001). Furthermore, two SAG deletion variants were recently identified, and the RING-H2 domain was found to be required for apoptosis protection (Sun, 1999). Here, we defined the genomic structure of the human $S A G$ gene and characterized a $S A G$ splicing variant $(S A G-v)$ and two pseudogenes ( $S A G P 1$ and $S A G P 2$ ) of the $S A G / R O C / R b x /$ Hrt family.

\section{MATERIALS AND METHODS}

\section{Computer database search}

The database was searched using BLAST (tblastn) from NCBI (Altschul et al., 1997). The sequences were from the 113 release of GenBank (Benson et al., 2000) and were clustered using the LEADS ${ }^{\mathrm{TM}}$ software from Compugen (Tel Aviv, Israel).

\section{Cloning of SAG-v and SAGP1}

Epitope-tagged SAG-v and SAGP1 were cloned by RT-PCR with muscle or heart mRNA (Clontech) as the template (Sun et al., 1993b). For FLAG-tagged SAG-v, the primers used were SAG-Bam-Flag01: 5'-CGCGGATCCGCCACCATGGACTACAAGGACGACGA TGACAAGGCCGACGTGGAAGACGGA-3' and SAG-VAR-Xh02: 5'-CCGCTCGAGCTACCGATGGTCATCCAGACA-3'. For HA-tagged SAG-v, the primers were SAG-HA01: 5'-CGCGGATCCGCCACCATGTATCCATATGATG TTCCAGATTATGCT GCCGACGTGGAAGAC-3' and SAG-VAR-Xh02. For HA-tagged SAGP1, the primers were SAG3-HA-Bam03: 5'-CGCGGATCCGCCACCATG T A T CCA T A T GA T G T T C CA GA T T A T GCTTTGTGGTTAATAACTGTGC-3' and SAG3-Xh02: 5'CCGCTCGAGTCACAAAACAGTTAAGCTTGATGG-3' that amplifies a cDNA fragment encoding 84 amino acids (translating from the second methionine). To validate SAGP1 EST sequences, RT-PCR direct sequencing (Sun et al., 1993a) was performed with primers SAG3-Bam1A: 5'-CGCGGATCCGGTAAAGTGTCCTGTGC-3' ${ }^{\prime}$ and SAG3-Xh02. To simultaneously amplify both SAG and SAG-v, the primers SAGBam-Flag01 and SAGV.02: 5'-GTAGGGCACTGGATTACAAG-3' were used. The PCR products were cloned into pcDNA3 and sequenced.

\section{Northern and Western blot analysis}

Northern analysis was performed as described previously (Sun et al., 1992). To determine SAG expression in transformed yeast cells, Western blot analysis was performed as described (Swaroop et al., 2000). Briefly, yeast cell extracts were pre- pared by vortexing the yeast with glass beads in the presence of urea/SDS lysis buffer supplemented with protease inhibitors. Cell extracts were then subjected to PAGE and transferred to a nylon membrane. The membrane was probed with HA-peroxidase antibody (Boehringer Mannheim) followed by incubation with enhanced chemiluminescence Western blotting detection reagents (Amersham) and autoradiography.

\section{Complementation, yeast sporulation, and dissection}

The HA-tagged human SAG and SAG-v were cloned into the yeast expression vector $\mathrm{p} 424-\mathrm{TRP}$ for complementation studies. The constructs were transformed into the heterozygous knockout strain (ySAG/ySAG::Kan) using the YeastMaker Transformation System from Clontech. Expression of SAG and SAG-v was detected by Western blotting using anti-HA antibody. The clones that expressed exogenous protein were sporulated and dissected as described previously (Swaroop et al., 2000).

\section{Transfection, metabolic labeling, and extract preparation}

Transient transfection studies were carried out in human $293 \mathrm{~T}$ cells grown in DMEM (GIBCO/BRL) containing heatinactivated FBS (GIBCO/BRL). Cells were cotransfected with pcDNA3 vectors expressing either HA-SAG or HA-SAG-v and cullin-1 plasmid by the calcium phosphate method (Sun et al., 1997). At $48 \mathrm{~h}$ post-transfection, cells were metabolically labeled for $2 \mathrm{~h}$ with Easy Tag Express $\left({ }^{35} \mathrm{~S}\right)$ Protein labeling mix $(100 \mu \mathrm{Ci} / \mathrm{ml})$ from NEN. Cell pellets were resuspended in 0.2 $\mathrm{ml}$ of buffer A (10 mM Tris $\mathrm{HCl}, \mathrm{pH} 7.4 ; 10 \mathrm{mM} \mathrm{NaCl}, 0.5 \%$ NP40, $1 \mathrm{mM}$ PMSF, antipain $2 \mu \mathrm{g} / \mathrm{ml}$, leupeptin $2 \mu \mathrm{g} / \mathrm{ml}$ ) and lysed by sonication. An equal volume of buffer B (20 mM Tris $\mathrm{HCl}, \mathrm{pH} 7.4 ; 1 \mathrm{M} \mathrm{NaCl}, 0.2 \% \mathrm{NP} 40,1 \mathrm{mM}$ PMSF, antipain 2 $\mu \mathrm{g} / \mathrm{ml}$, and leupeptin $2 \mu \mathrm{g} / \mathrm{ml}$ ) was added, and the resulting mixture was rocked for $1 \mathrm{~h}$ at $4^{\circ} \mathrm{C}$ prior to centrifugation at $100,000 \times g$ at $4^{\circ} \mathrm{C}$ for $1 \mathrm{~h}$.

\section{Immunoprecipitation}

The supernatant liquid prepared above was rocked with HA antibody (HA-7; Sigma) and protein agarose beads (40 $\mu \mathrm{l}$; Upstate Biotechnology) for $1 \mathrm{~h}$ at $4^{\circ} \mathrm{C}$. The beads were washed sequentially three times with $0.5 \mathrm{ml}$ of buffer $\mathrm{C}$ (buffer A and $\mathrm{B}$ mixed in equal volumes) and two times with $0.5 \mathrm{ml}$ of buffer D (25 mM Tris HCl, pH 7.4; 1 mM EDTA, 0.01\% NP40, 10\% glycerol, and $50 \mathrm{mM} \mathrm{NaCl}$ ). Bound proteins were released by boiling the beads in the presence of $20 \mu \mathrm{l}$ of SDS loading buffer. Half the reaction mixture was used for SDS-PAGE, followed by autoradiography.

\section{Ubiquitin labeling and Ub ligation assay}

The assay was performed as described previously (Swaroop et al., 2000). Protein kinase-ubiquitin (PK-Ub), prepared as a fusion protein with a protein kinase $\mathrm{C}$ recognition site (LRRASV), was kindly provided by Dr. Z. Pan at Mount Sinai School of Medicine, New York. The PK-Ub (7 $\mu \mathrm{g})$ was first labeled with ${ }^{32} \mathrm{P}$-ATP in a $20-\mu$ reaction mixture containing 20 $\mathrm{mM}$ Tris $\mathrm{HCl}, \mathrm{pH} 7.4 ; 12 \mathrm{mM} \mathrm{MgCl} 2,2 \mathrm{mM} \mathrm{NaF}, 50 \mathrm{mM} \mathrm{NaCl}$, $25 \mu \mathrm{M}$ ATP, $5 \mu \mathrm{Ci}$ of $\left[\gamma^{3}{ }^{32} \mathrm{P}\right]$-ATP, BSA $0.1 \mathrm{mg} / \mathrm{ml}$, and $1 \mathrm{U}$ of cAMP kinase (Sigma). The reaction mixture was incubated 
at $37^{\circ} \mathrm{C}$ for $30 \mathrm{~min}$, followed by heat inactivation of the kinase at $70^{\circ} \mathrm{C}$ for $3 \mathrm{~min}$. Immunoprecipitated samples, prepared as described above, were added to a Ub ligation reaction mixture (30 $\mu \mathrm{l})$ that contained $50 \mathrm{mM}$ Tris $\mathrm{HCl}, \mathrm{pH} 7.4 ; 5 \mathrm{mM} \mathrm{MgCl} 2,2$ $\mathrm{mM} \mathrm{NaF}, 10 \mathrm{nM}$ okadaic acid, $2 \mathrm{mM}$ ATP, $0.6 \mathrm{mM}$ DTT, $1 \mu \mathrm{g}$ of ${ }^{32} \mathrm{P}-\mathrm{Ub}, 60 \mathrm{ng}$ of E1 (Affiniti), and $300 \mathrm{ng}$ of mouse Cdc34 (E2; kindly provided by Dr. Z. Pan). The reaction mixture was incubated at $37^{\circ} \mathrm{C}$ for $1 \mathrm{~h}$ with constant shaking. The reaction mixture was then combined with $20 \mu \mathrm{l}$ of $4 \times$ Laemmli loading buffer, boiled for $3 \mathrm{~min}$, and subjected to $12.5 \%$ and $7.5 \%$ SDSPAGE analysis, followed by autoradiography.

\section{RESULTS}

\section{Genomic structure of human SAG/ROC2/Rbx2/Hrt2 gene}

We have previously reported the cloning of a 754-bp cDNA fragment (nt 1-342; the ORF; nt 343-754; the 3' UTR) that encode SAG, a redox-inducible protein and the second member of ROC/Rbx/Hrt family (Duan et al., 1999; Ohta et al., 1999; Swaroop et al., 2000). Using this fragment as a Northern probe, we determined that the size of SAG mRNA is about 800 bp (Duan et al., 1999). The $S A G$ gene was mapped to chromosome 3q22-24 (Duan et al., 1999). To define the genomic structure of SAG, we searched the GenBank database with this 754-bp cDNA fragment (Accession No. AF092878) as the bait. An 192$\mathrm{kb}$ chromosome 3 genomic clone, consisting of 35 unordered contigs (Accession No. AC067952.4), was shown to contain the
$S A G$ gene. Comparison of $S A G$ genomic and cDNA/protein sequences revealed that the wildtype $S A G$ consists of three exons (exons 1, 3, and 4) (Fig. 1; also see below). Exon 1 encodes the first 59 amino acids, whereas the rest of the sequence, including the RING-finger domain, is encoded by the exons 3 and 4 (Fig. 1). The exon-intron boundary sequences are shown in Table 1, and all introns meet the GT-AG rule, beginning with the GT and ending with the AG. The exact length of intron 1 cannot be determined because of a gap of unknown length in the sequence. Recently, $S A G$ was also cloned by others in a yeast two-hybrid screening and found to be a casein kinase IIbinding protein (Son et al., 1999). This SAG clone (Accession No. AF164679) consists of 836 bp with an additional 24 nt located at the $5^{\prime}$ end of the ATG and mapped to the same exon as the 175 residues downstream from the ATG (exon 1). Thus, on the basis of SAG mRNA size, we have analyzed the nearly full-length of $S A G$ cDNA and confirmed the $S A G$ chromosomal DNA sequences.

\section{Identification and cloning of a human SAG variant $(S A G-v)$}

The SAG/ROC/Rbx/Hrt family has two known members (Kamura et al., 1999; Ohta et al., 1999; Seol et al., 1999; Swaroop et al., 2000; Tan et al., 1999) that show high sequence similarity with Apc11, a component of the anaphase promoting complex (APC) (Yu et al., 1998; Zachariae et al., 1998). To identify other potential SAG family member(s), we searched a clustered EST database with known family members as the queries. A cluster of three ESTs (AA442424, AA749029, and N95727) gave rise to a SAG variant (SAG-v) that contains a

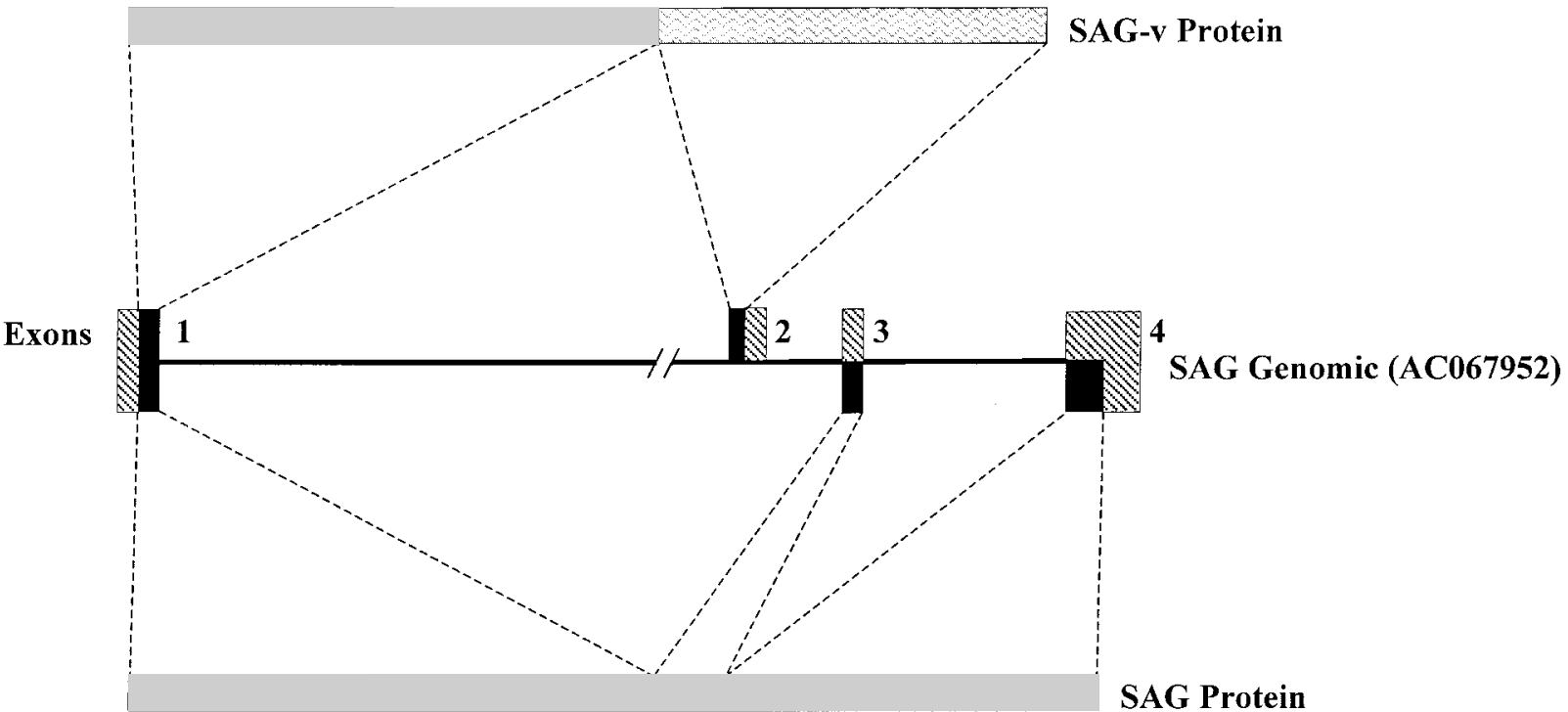

coding

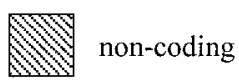

$\sim 1000 \mathrm{bp}$

FIG. 1. SAG genomic and protein structure. The exons are indicated by the solid boxes with their respective numbers. Introns are indicated by the thin line. The double slashes indicate the location of a gap between contigs. The hashed areas indicate regions that are transcribed but not translated. The shaded boxes represent the ORF of SAG (bottom) and SAG-v (top) proteins. The projection of protein domains onto their corresponding exons is indicated. Also indicated are the $\mathrm{C} 3 \mathrm{H} 2 \mathrm{C} 3 \mathrm{RING}$ finger residues. 
Table 1. Exon-Intron Boundary Sequences of SAG/ROC2/Rbx2/Hrt2

\begin{tabular}{|c|c|c|c|}
\hline Exons & Intron-exon-intron junctions & $S A G-v$ & AC067952.4 \\
\hline 1 & .... CAGGTGATGGgtaagcgetg & $1-175$ & $<36333-36507$ \\
\hline 2 & tttttacagATGAGGGAAT・...TTCTGTGTCAgtaagtatgg & $176-439$ & $\begin{array}{c}189836-190099 \\
\text { reverse }\end{array}$ \\
\hline 3 & 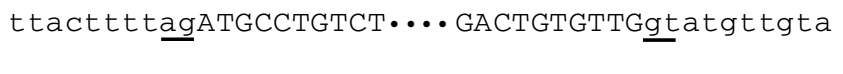 & $440-487$ & $\begin{array}{c}189187-189234 \\
\text { reverse }\end{array}$ \\
\hline 4 & tetcttcagTGGTCTGGGG... & 488-end & $\begin{array}{c}187398->187584 \\
\text { reverse }\end{array}$ \\
\hline
\end{tabular}

The exon sequences are shown in upper case, whereas the intron sequences are in lower case. Only the partial sequences of each exon and intron adjacent to the splice sites are shown. The conserved intron (starting sequence GT and ending sequence AG) is underlined. Because the exact 5' and $3^{\prime}$ ends of the SAG-v mRNA are not defined, $<$ and $>$ symbols are used to indicate that the sequence continues beyond the indicated numbering. Sequence positions in AC067952.4 are relative to the total clone numbering. Exons 2-4 are listed in reverse order from exon 1 because they fall within a separate contig of the AC067952 clone.

264-bp insertion at nucleotide 177 of the SAG ORF (Fig. 2A; underlined). The ORF of SAG-v predicts a protein of 108 amino acids with the first 59 amino acids identical to SAG and the remaining 49 amino acids a novel sequence at the $\mathrm{C}$ terminus. Because of inframe stop codons, the entire RING-H2 finger domain is not translated (Fig. 2B). Sequence comparison between $S A G-v$ and the $S A G$ genomic clone revealed a $100 \%$ match of a 264-bp insertion sequence with the genomic clone (not shown), indicating it belongs to an additional exon, not present in wildtype $S A G$. Thus, the genomic structure of $S A G$ consists of four exons and three introns. The $S A G-v$ consists of all four exons, whereas the wildtype $S A G$ contains exons 1, 3, and 4 with exon 2 spliced out. Shown in Figure 1 is the protein structure of SAG-v and SAG and their corresponding encoding exons. The coding sequences for SAG-v is encoded by exons 1 and exon 2, whereas that for SAG is encoded by exons 1,3 , and 4 (bottom part of the figure). The entire ORF of $S A G-v$ was cloned by RT-PCR using mRNA from human muscle and heart as the templates and was confirmed by DNA sequencing. The $S A G-v$ sequence has been deposited in GenBank with the Accession Number AF312226.

\section{Identification of two potential pseudogenes of SAG/ROC/Rbx/Hrt family}

Similar EST database mining identified a cluster of two ESTs (AI023768 and AA99464) that predicts an ORF of 84 amino acids (translated from the second methionine), termed SAGP1 (see below). Figure 3 shows a pile-up comparison among SAG family members. Five of the eight conserved $\mathrm{C} 3 \mathrm{H} 2 \mathrm{C} 3$ residues are preserved in $S A G P 1$, and the other two substitutions $(\mathrm{H} \rightarrow \mathrm{R}, \mathrm{C} \rightarrow \mathrm{T})$ seen in SAG-p are also seen in some RING-finger proteins with degeneracy (Boddy et al., 1994; Borden and Freemont, 1996). Thus, in SAGP1, the functional RING-finger domain appears to be conserved, and this clone may represent a third member of the SAG/ROC/Rbx/Hrt family. Direct RT-PCR sequencing of human heart mRNA using primers derived from the ESTs revealed an ORF of 112 codons with two inframe stop codons between the first and the second methionine (Fig. 3). The overall sequence identity of this clone is $36 \%$ with ROC1 and $30 \%$ with $S A G$. Because disruption of an ORF by stop condons is reminiscent of a pseudogene, this clone was named SAGPl (potential pseudogene of the $S A G / R O C / R b x / H r t$ family). Furthermore, a high-throughput genomic (HTG) database search revealed that $S A G P 1$ maps to human chromosome 5 (Accession No. AC012636). The genomic sequence analysis showed that the SAGPl gene is intronless, another characteristic of a pseudogene. As will be discussed below, $S A G P 1$ is not translated into protein in all expression vectors tested, further suggesting that it is a pseudogene of the $S A G / R O C / R b x / H r t$ family.

Similar HTG database searching revealed an additional pseudogene of the SAG family, designated SAGP2 (Accession No. AC016923). This sequence mapped to chromosome 3 and shared $88 \%$ sequence identity with $S A G$ and $47 \%$ identity with ROC1. As shown in Figure 3, all eight conserved $\mathrm{C} 3 \mathrm{H} 2 \mathrm{C} 3$ residues are preserved in SAGP2, except an $\mathrm{H} \rightarrow \mathrm{R}$ substitution at the fifth position of the RING domain. However, the $S A G P 2$ gene is intronless and contains two inframe stop codons, at positions 31 and 47, which well qualifies it as the second pseudogene of the $S A G / R O C$ family.

\section{Expression of SAG-v and SAGPI in human tissues and cell lines}

Using Image clone AA442424 (consisting of the sequence encoding a part of N-terminal SAG, 264-bp insertion sequence, and the sequence for the entire C-terminal RING domain) as a $S A G-v$ probe for Northern analysis, we detected the expression of $S A G$ as a 0.8 -bp band, but no expression of $S A G-v$ (expected to be 264 bp longer), in multiple human tissues (data not shown). The result suggested that expression of $S A G-v$, if any, is quite low. To increase detection sensitivity, we performed RT-PCR using the primers flanking the $S A G$ - $v$ ORF. As shown in Figure 4A, expression of $S A G-v$ was detected in human placenta, muscle, heart, and liver (both adult and fetal). Expression of $S A G-v$ was also detectable by RT-PCR in more than 20 human cell lines (data not shown).

To determine the relative expressions of $S A G$ and $S A G-v$, we performed RT-PCR using a pair of primers flanking the entire ORF of $S A G$. If $S A G-v$ is present as a 264-bp insertion variant, we should be able to detect a band with a size of 264 bp larger than wildtype $S A G$. Indeed, as shown in Figure 4B, in addition to a very abundant band corresponding to $S A G$, a faint band with a size corresponding to $S A G-v$ was detected and confirmed by Southern blot analysis (data not shown). 


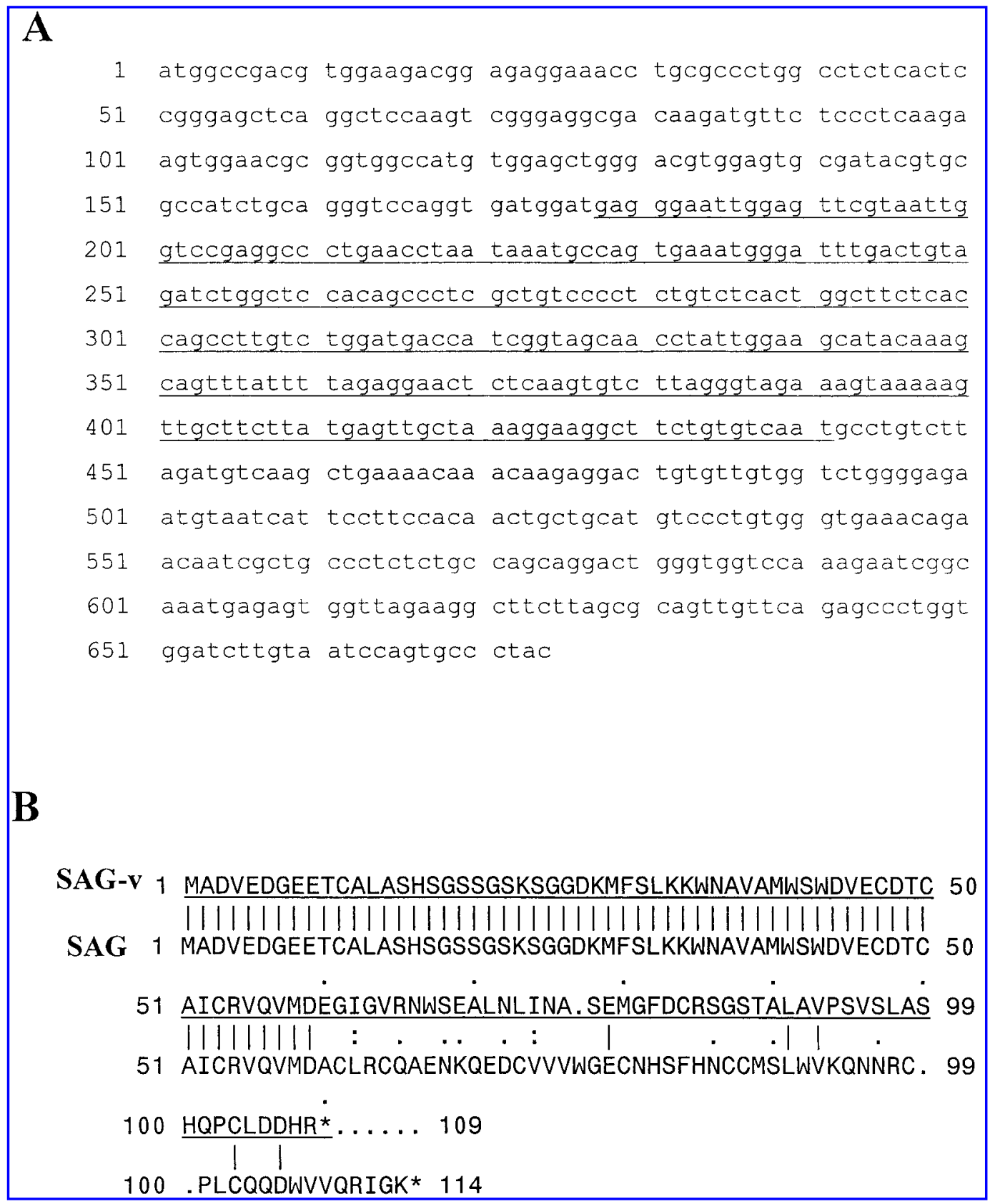

FIG. 2. cDNA sequence (A) and protein sequence (B) of SAG splicing variant, SAG-v. (A) Alternative splicing of SAG that retains exon 2 results in a 264-bp insertion in the wildtype SAG ORF. The inserted sequence is underlined. (B) Translation of SAG-v and its comparison with wildtype SAG. Because of inframe stop codons, SAG-v translation stops at codon 109, and the RING finger domain seen in wildtype SAG is not translated. The ORF of SAG-v is underlined.

Thus, both $S A G$ and $S A G-v$ are expressed in human muscle and heart, but $S A G$ expression is much higher than that of $S A G-v$.

We have also examined $S A G P 1$ expression in multiple human tissues by Northern analysis using the SAGP1 ORF (a fragment encoding 84 amino acids) as the probe. As shown in Figure 5, SAGP1 was highly expressed in heart and muscle, moderately expressed in kidney, liver, and placenta (Fig. 5 ; top panel). The expression pattern is very similar to that of SAG (Duan et al., 1999) and ROC1 (middle panel). Thus, although $S A G P 1$ appears to be a pseudogene, it is indeed expressed, as is also true of some other pseudogenes (Mighell et al., 2000). It remains to be determined whether SAGP2 is also expressed. Because of its high sequence homology with $S A G(88.5 \%$ identity at the protein level and $91 \%$ identity at the nucleotide level), it is anticipated that the $S A G P 2$ probe will cross-react with $S A G$ mRNA, which could interfere with the analysis. 


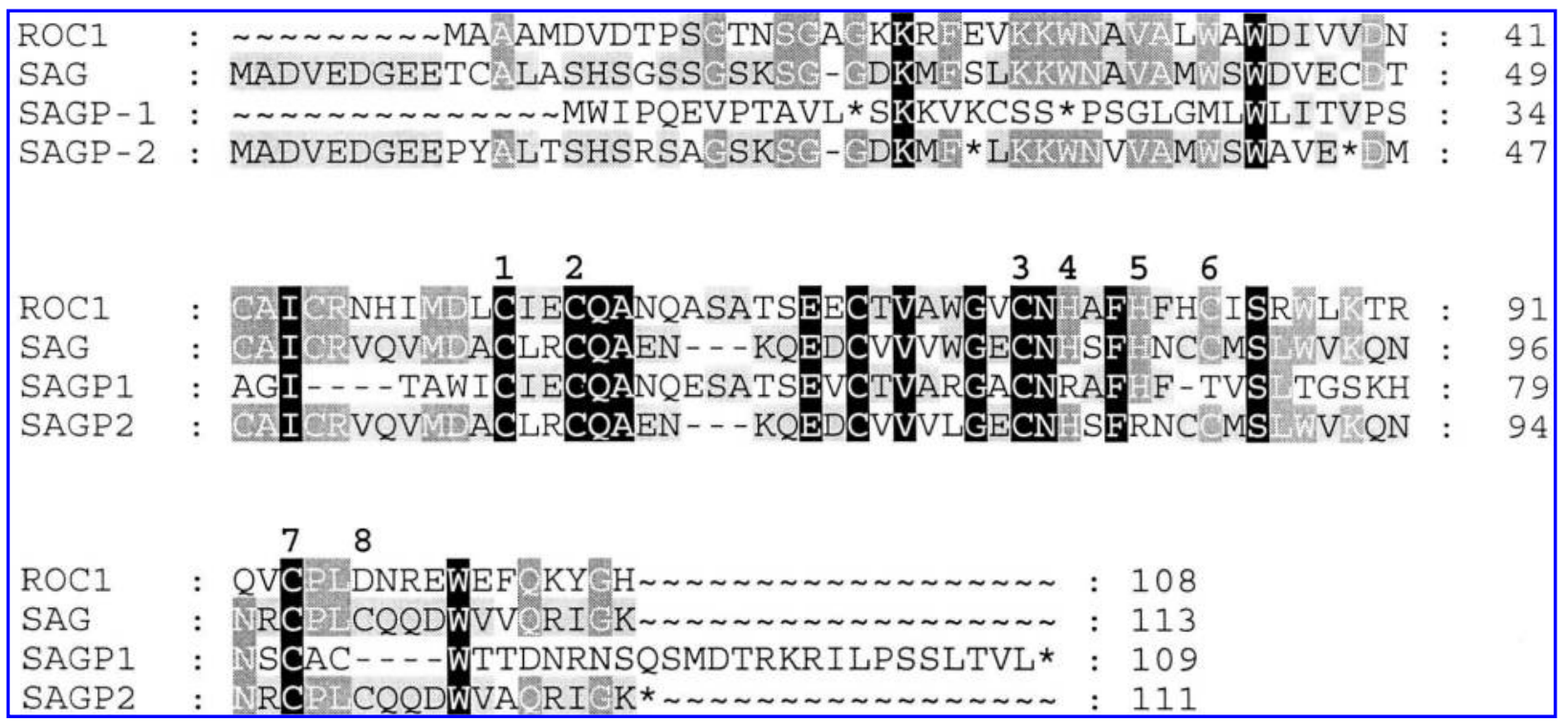

FIG. 3. Comparison of ROC1 and SAG/ROC2 with two SAG family pseudogenes, SAGP1 and SAGP2. For SAGP1, two ESTs (AI023768 and AA699464) were identified through a database search using ROC/SAG as queries. The sequence shown here was confirmed by RT-PCR direct sequencing. For SAGP2, a genomic coding (AC016923) was identified through a HTG database search using a 754-bp SAG cDNA as a query. Translation was initiated from the first ATG for protein sequence comparison purposes. The numbers (1-8) above the amino acid codons indicate the residues forming the C3H2C3 RING finger domain. Identical residues in all four sequences are blackened, and identical residues in three sequences are shaded.
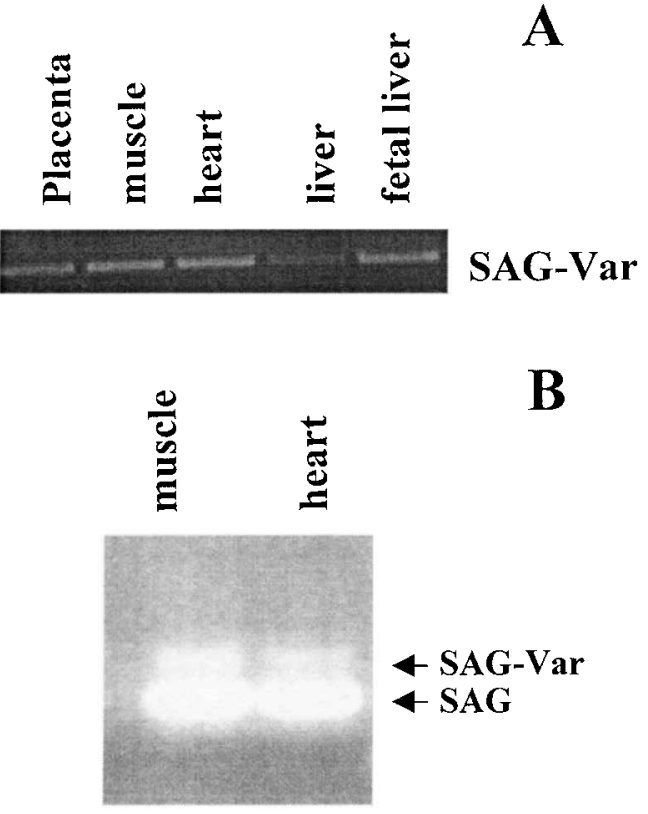

FIG. 4. Expression of SAG-v in human tissues. Total RNA from the indicated human tissues was subjected to RT-PCR using SAG-v-specific primers (note that the downstream primer is located within the insertion sequence)flanking the entire ORF (A) or specific primers for wildtype SAG that amplify both SAG and SAG-v (B), as detailed in Materials and Methods. The PCR fragments corresponding to SAG or SAG-v are indicated and were confirmed by Southern analysis.
Unlike SAG, SAG-v does not rescue death phenotype induced by ySAG disruption

We have previously shown that yeast $S A G$ is essential for growth and that disruption of the gene leads to cell death, which can be rescued by wildtype human $S A G$ but not by $S A G$ mutants (Swaroop et al., 2000). To test the potential rescuing activity of $S A G-v$, heterozygous ySAG knockout cells (ySAG/ySAG::Kan) were transformed with $S A G-v$ cloned into the yeast expression vector p424 (carrying a tryptophan selectable marker) and also with wildtype $S A G$ control. Transformants were selected by plating on tryptophan minus medium, and expression was confirmed by Western blot analysis with HA antibody. Figure 6A shows two individual clones that expressed wildtype $S A G$ or $S A G-v$, respectively, at a compatible level. These clones were sporulated and dissected. Rescue of ySAG::kan cells should result in four viable haploids displaying three possible genotypes: wildtype (grown only in YPD medium), wildtype + hSAG-v plasmid (grown in YPD minus tryptophan), and ySAG::kan + hSAG-v plasmid (grown in YPD-G418-containing medium). The tetrad dissected from $S A G$ - $v$-transformed ySAG/ySAG::kan cells gave rise to two viable haploids that grew only in YPD medium (Fig. 6B). This result strongly suggests that all viable haploids contain wildtype ySAG, an idea that was confirmed by PCR analysis (data not shown). Four viable haploids were seen in the tetrad dissected from wildtype $S A G$-transformed cells (Figure 6C) with the expected genotypes (Swaroop et al., 2000). Thus, unlike wildtype $S A G, S A G-v$ is unable to rescue the death phenotype induced by ySAG disruption. A similar yeast rescue assay was performed with a $S A G P 1$ construct (translated from the second methionine to give rise to an 84 -amino acid protein). No ex- 


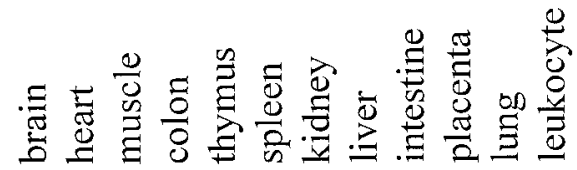

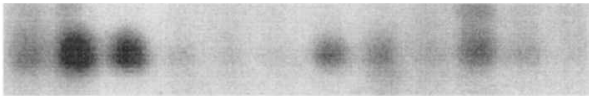

SAG-p

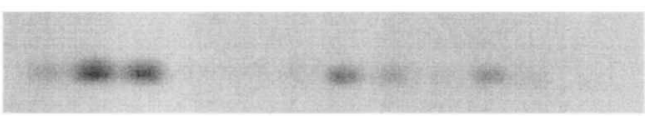

ROC1

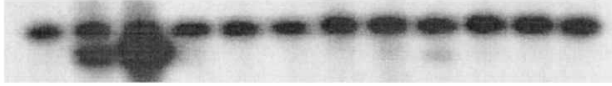

Actin

FIG. 5. Expression of $S A G P 1$ and $R O C 1$ in multiple human tissues. A membrane blotted with $1 \mu \mathrm{g}$ of poly(A) ${ }^{+}$mRNA from indicated human tissues (ClonTech) was subjected to Northern analysis, probed with SAG-p cDNA (top), ROC1 cDNA (middle), and $\beta$-actin (bottom) as described in Materials and Methods.

pression of SAGP1 protein could be detected in transformed yeast cells.

SAG-v does not bind to cullin-1 and has no ligase activity

We have recently shown that, like ROC1/Rbx1/Hrt1, SAG binds to cullin-1 and has associated E3 ubiquitin ligase activity (Swaroop et al., 2000). We also found that E3 ubiquitin ligase activity of SAG/cullin-1 complex is required for the rescue of the death phenotype induced by ySAG disruption (Swaroop et al., 2000). To further define the biochemical nature of SAG-v, we examined its potential ability to bind to cullin-1 and its E3 ubiquitin ligase activity. The $S A G-v$ gene was cotransfected with cullin-1, along with vector/cullin-1 and SAG/cullin-1 cotransfection controls, into 293 cells, followed by ${ }^{35} \mathrm{~S}$-Met labeling, immunoprecipitation, and ligase activity assay. Because expression of $S A G-v$ was much less than that of $S A G$ after transfection, as measured by direct Western blot (data not shown), a fourfold excess of cell lysate from $S A G$ - $v$-transfected cells was used for immunoprecipitation.

As shown in Figure 7A, the level of SAG-v and SAG proteins was comparable after adjustment for the input protein. Unlike SAG, which binds to cullin-1 (lane 3, Fig. 7A), SAG-v was found not to bind with cullin-1 (Fig. 7A). It is noteworthy that because of the fourfold excess of protein used in the SAG-v sample for the assay, some nonspecific binding bands were evident (lane 2). We also measured potential ligase activity of SAG-v compared with ROC1 and SAG. As shown in Figure 7B, ROC1 and SAG had E3 ubiquitin ligase activity and induced ubiquitin polymer formation (lanes 1 and 2), whereas SAG-v had very little, if any, such activity (lane 3 ). Thus, biochemically, SAG-v differs from wildtype SAG. The lack of ligase activity of SAG-v is most likely attributable to the absence of the RING-finger domain required for this activity.

We have attempted to express $S A G P 1$ using constructs that would initiate protein translation from either the first methionine (containing inframe stop codons between two methionines see Fig. 3) or the second methionine (encoding an 84-amino acid protein). Both in vitro transcription-coupled translation and in vivo human kidney 293 cell transfection assay showed no protein expression from either construct (data not shown), further confirming that $S A G P 1$ is a pseudogene of the $S A G / R O C$ family. Similar analysis has not been performed with $S A G P 2$.

\section{DISCUSSION}

The SAG protein is evolutionarily conserved (for details, see review by Sun et al., 2001). Two members of the

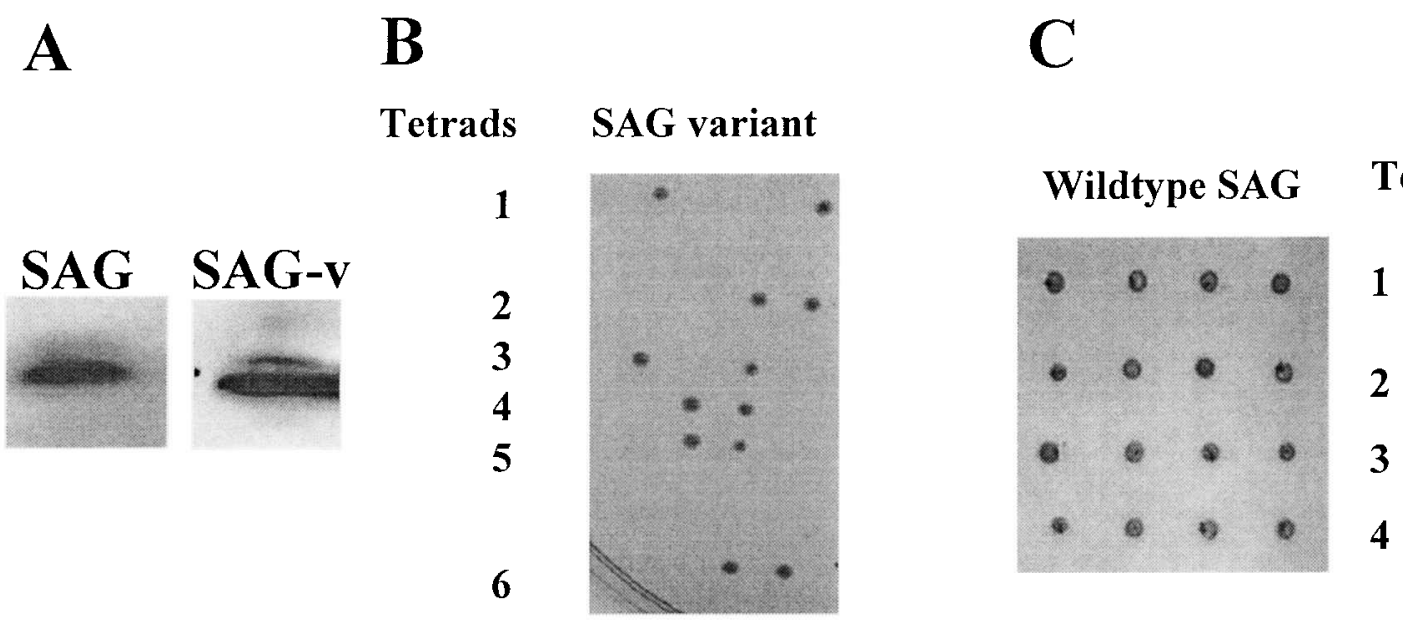

FIG. 6. SAG-v failed to rescue yeast death phenotype induced by SAG disruption. Heterozygous ySAG knockout cells (ySAG/ySAG::kan) were transformed with an $h S A G$ - or $S A G$ - $v$-expressing vector (p424) containing TRP as a selectable marker. Transformants were then selected on a TRP-minus plate. Several surviving clones were measured for SAG or SAG-v expression by Western blot. (A) Expression of SAG or SAG-v protein in two representative clones. These two clones were sporulated. Six independent tetrads from SAG-v (B) and four from SAG $(\mathbf{C})$, as the control, were dissected and grown in YPD medium. 


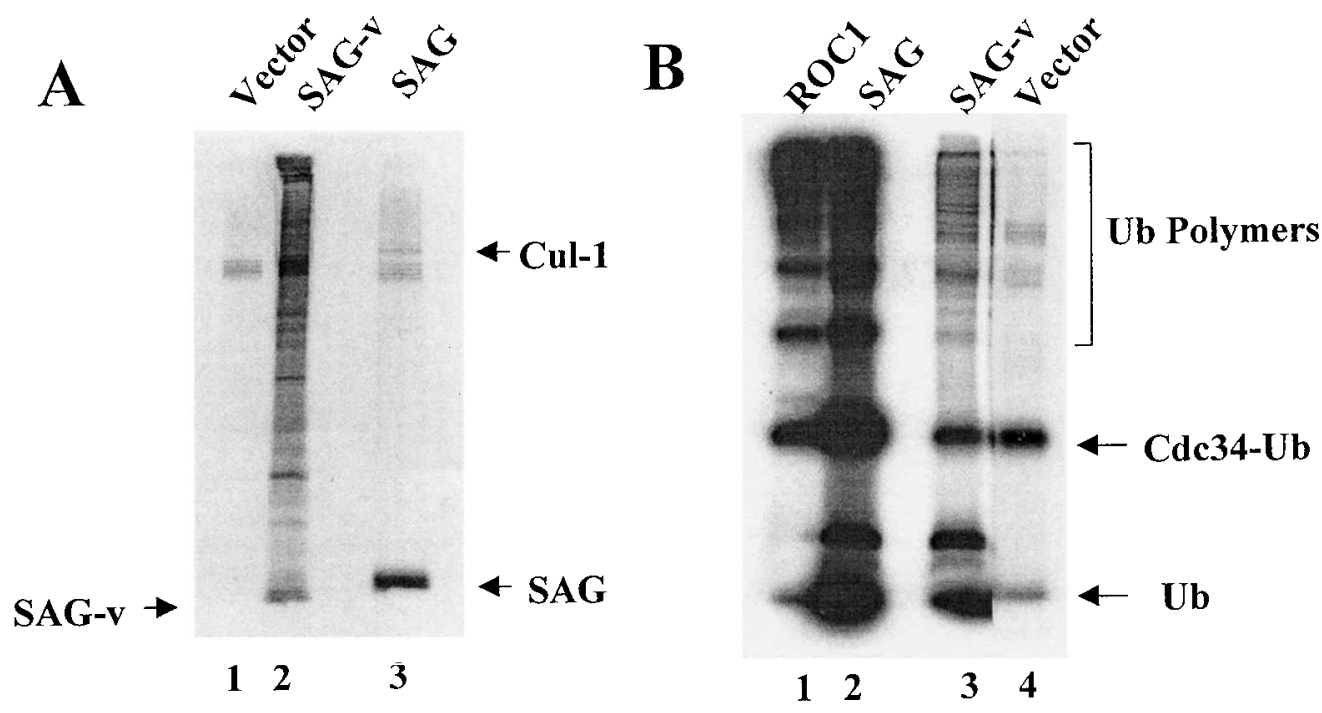

FIG. 7. SAG-v does not bind to Cull and has no ubiquitin ligase activity. (A) SAG and SAG-v expression and binding to cullin-1. HA-tagged SAG or HA-tagged SAG-v or the empty vector control was cotransfected with cullin-1 into 293 cells, followed by ${ }^{35} \mathrm{~S}$-methionine labeling. Cell lysates were immunoprecipitated with HA-tag antibody, followed by gel electrophoresis and autoradiography. About fourfold excess of cell lysates was used for SAG-v to ensure an approximately equal level of SAG expression. (B) E3 ubiquitin ligase activity of ROC1 and SAG but not SAG-v. HA-tagged ROC1, wildtype SAG, SAG-v, or the empty vector control was cotransfected with cullin- 1 into 293 cells, followed by ${ }^{35} \mathrm{~S}$-methionine labeling. Cell lysates (with approximately equal amount of SAG and SAG-v protein) were immunoprecipitated with HA-tag antibody, followed by ligase activity assay, as detailed in Materials and Methods.

SAG/ROC1 family have been cloned and characterized in humans. They are SAG (Accession No. AF092878) and ROC1 (Accession No. AF142059) (Duan et al., 1999; Kamura et al., 1999; Ohta et al., 1999; Seol et al., 1999; Tan et al., 1999). Although $S A G$ and $R O C 1$ share only $53 \%$ overall sequence identity, seven of eight cysteine/histidine residues that constitute the RING-finger domain are identical, and, importantly, they are functionally equivalent. Although uncharacterized, computer analysis revealed two additional family members: a hepatocellular carcinoma-associated RING-finger protein (Accession No. AF247565) and a hypothetical protein (Accession No. NP_057560 or AF151048). The SAG and ROC1 family is highly evolutionarily conserved across different species. A BLAST search identified two family members in mouse (Accession Nos. AF140599 and AF092877) with sequence identity of $100 \%$ with human ROC1 and $96 \%$ with human $S A G$ and four members in Drosophila melanogaster (Accession Nos. AAF45536, T13388, AAF47382, and AAF52694) with sequence identity ranging from $37 \%$ to $91 \%$. In Caenorhabditis elegans, there are three members (Accession Nos. T27823, T29620, and $\mathrm{T} 21802$ ) with a sequence identity ranging from $35 \%$ to $71 \%$. One family member was identified in the fission yeast Schizosaccharomyces pombe (Accession No. Z98977; 87\% with $R O C 1$ and $59 \%$ with $S A G$ ), in budding yeast Saccharomyces cerevisiae (Accession No. NP_014508; 66\% with ROC 1 and 55\% with $S A G$ ), in Arabidopsis thaliana (Accession No. CAB87200; $81 \%$ with $R O C 1$ and $51 \%$ with $S A G$ ); and in Plasmodium falciparum (Accession No. T18513; 68\% with $R O C 1$ and $44 \%$ with $S A G)$. In addition, ROC1 is ho- mologous to the APC11 subunit of the APC/cyclosome (APC/C) (Zachariae et al., 1998). An Apc11 homolog is also found in yeast (Accession No. Z74056), Drosophila (Accession No. CAB63945), and possibly in C. elegans (Accession No. CAA86328).

Although $S A G$ has been well characterized biochemically and biologically (Duan et al., 1999, 2001; Huang et al., 2001; Sun, 1999, 2000; Sun et al., 2001; Swaroop et al., 1999, 2000; Yang et al., 2001), the genomic structure, splicing variant, and pseudogenes of $S A G$ have not been reported. Here, we have defined the genomic structure of $S A G$ that consists of four exons and three introns with exon 2 being spliced out in wildtype $S A G$. In addition to identify two pseudogenes, $S A G P 1$ and $S A G P 2$ of the $S A G$ family, we have also characterized a $S A G$ splicing variant, $S A G-v$, whose mRNA contains all four exons, but whose protein translation terminated at exon 2 because of several inframe stop codons. The $S A G$ $v$ gene was found to be expressed at a much lower level than wildtype $S A G$ in multiple human tissues. Because of its lack of a RING-finger domain, SAG-v has no E3 ubiquitin ligase activity and fails to complement yeast SAG. The biologic significance of $S A G-v$ is unknown at the present time. It appears unlikely that $S A G-v$ acts in a dominant-negative manner to inhibit SAG E3 ubiquitin ligase, as SAG-v does not bind to cullin-1. Thus, it would not compete with SAG for cullin-1 binding. However, we cannot exclude the possibility that SAG-V would compete with SAG for binding to other SCF components. Future study should be directed to identifying the SAG-v binding partners in an attempt to understand its biologic function. 


\section{ACKNOWLEDGMENTS}

We thank Dr. Zhen-Qiang Pan of the Mount Sinai School of Medicine for his help in setting up the ubiquitin ligase assay and for providing assay reagents and Dr. Yue Xiong at University of North Carolina at Chapel Hill for providing the Cul1 plasmid.

\section{REFERENCES}

ALTSCHUL, S.F., MADDEN, T.L., SCHAFFER, A.A., ZHANG, J., ZHANG, Z., MILLER, W., and LIPMAN, D.J. (1997). Gapped BLAST and PSI-BLAST: A new generation of protein database search programs. Nucleic Acids Res. 25, 3389-3402.

BAI, C., SEN, P., HOFMANN, K., MA, L., GOEBL, M., HARPER, J.W., and ELLEDGE, S.J. (1996). SKP1 connects cell cycle regulators to the ubiquitin proteolysis machinery through a novel motif, the F-box. Cell 86, 263-274.

BENSON, D.A., KARSCH MIZRACHI, I., LIPMAN, D.J., OSTELL, J., RAPP, B.A., and WHEELER, D.L. (2000). GenBank. Nucleic Acids Res. 28, 15-18.

BODDY, M.N., FREEMONT, P.S., and BORDEN, K.L. (1994). The p53-associated protein MDM2 contains a newly characterized zincbinding domain called the RING finger. Trends Biochem Sci. 19, 198-199.

BORDEN, K.L., and FREEMONT, P.S. (1996). The RING finger domain: A recent example of a sequence-structure family. Curr. Opin. Struct. Biol. 6, 395-401.

CARRANO, A.C., EYTAN, E., HERSHKO, A., and PAGANO, M. (1999). SKP2 is required for ubiquitin-mediated degradation of the CDK inhibitor p27. Nature Cell Biol. 1, 193-199.

CIECHANOVER, A. (1998). The ubiquitin-proteosome pathway: On protein death and cell life. EMBO J. 17, 7151-7160.

DUAN, H., TSVETKOV, M., LIU, Y., SONG, Y., SWAROOP, M., WEN, R., KUNG, H.-F., ZHANG, H., and SUN, Y. (2001). Promotion of the S-phase entry and cell growth under serum starvation by $\mathrm{SAG} / \mathrm{ROC} 2 / \mathrm{Rbx} 2 / \mathrm{Hrt} 2$, an E3 ubiquitin ligase component: Association with inhibition of p 27 accumulation. Mol. Carcinogen. 30, $37-46$.

DUAN, H., WANG, Y., AVIRAM, M., SWAROOP, M., LOO, J.A., BIAN, J., TIAN, Y., MUELLER, T., BISGAIER, C.L., and SUN, Y. (1999). SAG, a novel zinc RING finger protein that protects cells from apoptosis induced by redox agents. Mol. Cell. Biol. 19, 3145 3155.

FELDMAN, R.M., CORRELL, C.C., KAPLAN, K.B., and DESHAIES, R.J. (1997). A complex of Cdc4p, Skp1p, and Cdc53p/cullin catalyzes ubiquitination of the phosphorylated CDK inhibitor Sic1p [see comments]. Cell 91, 221-230.

HUANG, Y., DUAN, H., and SUN, Y. (2001). Elevated expression of $\mathrm{SAG} / \mathrm{ROC} 2 / \mathrm{Rbx} 2 / \mathrm{Hrt} 2$ in human colon cancer carcinomas: SAG does not induce neoplastic transformation, but its antisense transfection inhibits tumor cell growth. Mol. Carcinogen. 30, $62-70$.

KAMURA, T., KOEPP, D.M., CONRAD, M.N., SKOWYRA, D., MORELAND, R.J., ILIOPOULOS, O., LANE, W.S., KAELIN, W.G., ELLEDGE, S.J., CONAWAY, R.C., HARPER, J.W., and CONAWAY, J.W. (1999). Rbx1, a component of the VHL tumor suppressor complex and SCF ubiquitin ligase. Science 284, 657-661.

KING, R.W., DESHAIES, R.J., PETERS, J.M., and KIRSCHNER, M.W. (1996). How proteolysis drives the cell cycle. Science 274, 1652-1659.

MIGHELL, A.J., SMITH, N.R., ROBINSON, P.A., and MARKHAM, A.F. (2000). Vertebrate pseudogenes. FEBS Lett. 468, 109-114.

OHTA, T., MICHEL, J.J., SCHOTTELIUS, A.J., and XIONG, Y. (1999).
ROC1, a homolog of APC11, represents a family of cullin partners with an associated ubiquitin ligase activity. Mol. Cell 3, 535-541.

SEOL, J.H., FELDMAN, R.M.R., ZACHARIAE, W.Z., SHEVCHENKO, A., CORRELL, C.C., LYAPINA, S., CHI, Y., GALOVA, M., CLAYPOOL, J., SANDMEYER, S., NASMYTH, K., SHEVCHENKO, A., and DESHAIES, R.J. (1999). Cdc53/cullin and the essential Hrt1 RING-H2 subunit of SCF define a ubiquitin ligase module that activates the E2 enzyme Cdc34. Genes Dev. 13, 1614-1626.

SKOWYRA, D., CRAIG, K.L., TYERS, M., ELLEDGE, S.J., and HARPER, J.W. (1997). F-box proteins are receptors that recruit phosphorylated substrates to the SCF ubiquitin-ligase complex [see comments]. Cell 91, 209-219.

SKOWYRA, D., KOEPP, D.M., KAMURA, T., CONRAD, M.N., CONAWAY, R.C., CONAWAY, J.W., ELLEDGE, S.J., and HARPER, J.W. (1999). Reconstitution of G1 cyclin ubiquitination with complexes containing $\mathrm{SCF}^{\mathrm{Grr} 1}$ and Rbx 1. Science 284, 662-665.

SON, M.Y., PARK, J.W., KIM, Y.S., KANG, S.W., MARSHAK, D.R., PARK, W., and BAE, Y.S. (1999). Protein kinase CKII interacts with and phosphorylates the SAG protein containing ring-H2 finger motif. Biochem. Biophys. Res. Comm. 263, 743-748.

SUN, Y. (1999). Alteration of SAG mRNA in human cancer cell lines: Requirement for the RING finger domain for apoptosis protection. Carcinogenesis 20, 1899-1903.

SUN, Y. (2000). Identification and characterization of genes responsive to apoptosis: Application of DNA chip technology and mRNA differential display. Histol. Histopathol. 15, 1271-1284.

SUN, Y., BIAN, J., WANG, Y., and JACOBS, C. (1997). Activation of 553 transcriptional activity by 1,10-phenanthroline, a metal chelator and redox sensitive compound. Oncogene 14, 385-393.

SUN, Y., HEGAMYER, G., and COLBURN, N.H. (1993a). PCR-direct sequencing of a GC-rich region by inclusion of $10 \%$ DMSO: Application of mouse c-jun. BioTechniques 15, 372-374.

SUN, Y., NAKAMURA, K., HEGAMYER, G., DONG, Z., and COLBURN, N. (1993b). No point mutation of Ha-ras or p53 genes expressed in preneoplastic-to-neoplastic progression as modeled in mouse JB6 cell variants. Mol. Carcinogen. 8, 49-57.

SUN, Y., POMMIER, Y., and COLBURN, N.H. (1992). Acquisition of a growth-inhibitory response to phorbol ester involves DNA damage. Cancer Res. 52, 1907-1015.

SUN, Y., TAN, M., DUAN, H., and SWAROOP, M. (2001). SAG/ROC/Rbx/Hrt, a zinc RING finger gene family: Molecular cloning, biochemical properties and biological functions. Antioxidants Redox Signal. (in press).

SUTTERLUTY, H., CHATELAIN, E., MARTI, A., WIRBELAUER, C., SENFTEN, M., MULLER, U., and KREK, W. (1999). p45SKP2 promotes p27Kip 1 degradation and induces $S$ phase in quiescent cells. Nature Cell Biol. 1, 207-214

SWAROOP, M., BIAN, J., AVIRAM, M., DUAN, H., BISGAIER, C.L., LOO, J.A., and SUN, Y. (1999). Expression, purification, and biochemical characterization of SAG, a RING finger redox sensitive protein. Free Radicals Biol. Med. 27, 193-202.

SWAROOP, M., WANG, Y., MILLER, P., DUAN, H., JATKOE, T., MADORE, S., and SUN, Y. (2000). Yeast homolog of human $\mathrm{SAG} / \mathrm{ROC} 2 / \mathrm{Rb} 2 / \mathrm{Hrt} 2$ is essential for cell growth, but not for germination: Chip profiling implicates its role in cell cycle regulation. Oncogene 19, 2855-2866.

TAN, P., FUCHS, S.Y., CHEN, A., WU, K., GOMEZ, C., RONAI, Z., and PAN, Z.-Q. (1999). Recruitment of a ROC1-CUL1 ubiquitin ligase by Skp1 and HOS to catalyze the ubiquitination of IkBa. Mol Cell 3, 527-533.

TSVETKOV, L.M., YEH, K.-H., LEE, S.-J., SUN, H., and ZHANG, H. (1999). p $27^{\mathrm{kip} 1}$ ubiquitination and degradation is regulated by the SCF ${ }^{\text {skp2 }}$ complex through phosphorylated Thr187 in p27. Curr. Biol 9, 661-664.

YANG, G.-Y., PANG, L., GE, H.L., TAN, M., YE, W., LIU, X.-H., 
HUANG, F.-P., WU, D.-C., CHE, X.-M., SONG, Y., WEN, R., and SUN, Y. (2001). Attenuation of ischemia-induced mouse brain injury in mice by SAG, a redox inducible antioxidant protein. J. Cerebral Blood Flow Metab. 21, 722-733.

YU, H., PETERS, J.M., KING, R.W., PAGE, A.M., HIETER, P., and KIRSCHNER, M.W. (1998). Identification of a cullin homology region in a subunit of the anaphase-promoting complex. Science 279, 1219-1222.

ZACHARIAE, W., SHEVCHENKO, A., ANDREWS, P.D., CIOSK, R., GALOVA, M., STARK, M.J., MANN, M., and NASMYTH, K. (1998). Mass spectrometric analysis of the anaphase-promotiong complex from yeast: Identification of a subunit related to cullins. Science 279, 1216-1219.
Address reprint requests to: Dr. Yi Sun

Cancer Molecular Sciences Pfizer Global Research and Development Ann Arbor Laboratories 2800 Plymouth Road Ann Arbor, MI 48105

E-mail: yi.sun@pfizer.com

Received for publication April 18, 2001; accepted May 14, 2001. 
This article has been cited by:

1. Jiyi Yin, Maria F Chen, Terri H Finkel. 2004. Differential gene expression during HIV-1 infection analyzed by suppression subtractive hybridization. AIDS 18:4, 587-596. [CrossRef]

2. Yi Sun, Mingjia Tan, Hangjun Duan, Manju Swaroop . 2001. SAG/ROC/Rbx/Hrt, a Zinc RING Finger Gene Family: Molecular Cloning, Biochemical Properties, and Biological FunctionsSAG/ROC/Rbx/Hrt, a Zinc RING Finger Gene Family: Molecular Cloning, Biochemical Properties, and Biological Functions. Antioxidants \& Redox Signaling 3:4, 635-650. [Abstract] [PDF] [PDF Plus] 